\title{
Nueva estrategia para la gestión de calidad: implementación de cartas de servicios en una entidad pública peruana
}

\author{
Joseph Ugarte Mel \\ Consultor en Gestión de Calidad, Perú. \\ Correo electrónico: josephugartemel@gmail.com \\ Recibido: 23 de abril de 2015 / Aprobado: 23 de mayo de 2015
}

\begin{abstract}
Resumen: En el contexto de la modernización del Estado peruano, las entidades y gestores públicos han visto la necesidad de optimizar los servicios que ofrecen a la ciudadanía, demandando proyectos de corto plazo y alto impacto. En ese sentido, este artículo tiene el objetivo de promover las cartas de servicios como una herramienta de mejora continua para acercar e involucrar al ciudadano, estableciendo una relación de atención directa a través de compromisos; y para promover una gestión ágil, económica y con resultados inmediatos. Este producto innovador está alineado al sistema de gestión de calidad y ha generado un significativo impacto.
\end{abstract}

Palabras clave: cartas de servicios / gestión de calidad / nueva estrategia / administración pública

\section{New Strategy for Quality Management: Service Charters Implementation in a Peruvian Public Entity}

ABstRaCT: In the context of the Peruvian state modernization, entities and public managers have seen the need to optimize the services offered to citizens, currently demanding, short-term and high impact projects. In that sense, this article aims to disseminate Service Charters as a continuous improvement tool to approach and involving the citizen, establishing a direct care relationship through commitments and to promote agile, economic and immediate results management. This innovative product is aligned to the quality management system and has a significant impact.

Key words: service charters / quality management / new strategy / public administration 


\section{INTRODUCCIÓN}

La modernización del Estado peruano requiere que sus instituciones y gestores públicos sean capaces de optimizar los servicios que se brindan a la ciudadanía.

Debido a que los ciudadanos tienen necesidades concretas y expectativas que han de ser satisfechas, las administraciones de muchos países vienen utilizando instrumentos que permiten manifestar públicamente la materialización de los principios de transparencia, participación, responsabilidad y compromiso en la prestación de los servicios públicos.

A continuación se narra una experiencia inspirada en la metodología que siguió una organización pública peruana, en respuesta a la problemática nacional en la prestación de sus servicios, y que puede servir de modelo a otras instituciones.

\section{PROBLEMÁTICA}

En toda entidad pública como privada existen deficiencias que suponen problemas para la organización. Muchas veces el diseño y enfoque operacional de los servicios que ofrece una institución incorporan solo propósitos de gestión orientados al cumplimiento de las obligaciones formales determinadas por la normativa, carentes de canales y protocolos de atención, colaboración y coordinación con los usuarios del servicio, lo cual deriva en:

- desconocimiento del grado de satisfacción de los usuarios respecto de los servicios que brinda la institución

- procedimientos no formalizados ni estandarizados

- desconocimiento del proceso de atención por parte del usuario

- demora en la atención del servicio

- ausencia de canales de participación con el usuario

Estas deficiencias, una vez identificadas, son planteadas como oportunidades de mejora y yacen vulnerables a la experiencia y conocimientos de los especialistas en la materia, que de antemano citan una gama de herramientas que permiten lograr su desarrollo como tal.

En el peor de los escenarios, una organización con limitaciones presupuestales, con personal que se resiste al cambio y con dificultades 
burocráticas, debe idear estrategias que le permitan obtener logros a corto plazo, que generen un alto impacto en la organización, y que signifiquen un éxito institucional y personal en el camino a la excelencia.

Siguiendo la metodología de Deming ${ }^{1}$ y la matriz de dificultad versus impacto, una organización pública víctima de limitaciones como las mencionadas en el párrafo previo, pero con un equipo humano comprometido y bien dirigido, puede tomar las riendas de la modernización del Estado peruano y emprender el virtuoso viaje de la mejora continua y gestión de la calidad. Por ejemplo, en su afán de lograr la excelencia en la atención al usuario, el Servicio de Administración Tributaria (SAT) y el Organismo Supervisor de la Inversión en Energía y Minería (Osinergmin) han implementado el Sistema de Gestión de Calidad ISO 9001:2008. De igual manera, la Presidencia del Consejo de Ministros (PCM), a través de la Secretaría de Gestión Pública, viene impulsando la mejora de la atención al usuario y gestión por procesos, a través de normas y metodologías de progresiva y obligatoria implementación ${ }^{2}$.

\section{METODOLOGÍA}

Muchas de las deficiencias latentes en una organización son transversales a varios procesos. En ese sentido, es necesario identificar y mapear los procesos de atención al público más impactantes para el usuario, y a partir de ello seguir una metodología que permita a la organización obtener resultados tangibles a corto plazo. La implementación de cartas de servicios se convierte en una eficaz herramienta para lograrlo.

Según la Norma UNE 93200:2008, se entiende por carta de servicios el documento escrito por medio del cual las organizaciones informan públicamente a los usuarios acerca de los servicios que gestionan, los compromisos de calidad en su prestación, así como los derechos y obligaciones que les asisten. Para lograr su correcta implementación y mejora continua, se requiere cumplir detenidamente con ciertas etapas, conforme se muestra en la figura 1.

1 Edwards Deming, creador del ciclo de Deming, o el también conocido círculo PDCA, por sus siglas en inglés (Plan, Do, Check, Act). A través de estos cuatro pasos, esta estrategia pretende lograr la mejora continua en una organización.

2 Normas como la "Estrategia de modernización" y el "Manual para mejorar la atención a la ciudadanía en las entidades de la administración pública”, entre otras, han sido aprobadas desde 2012. 


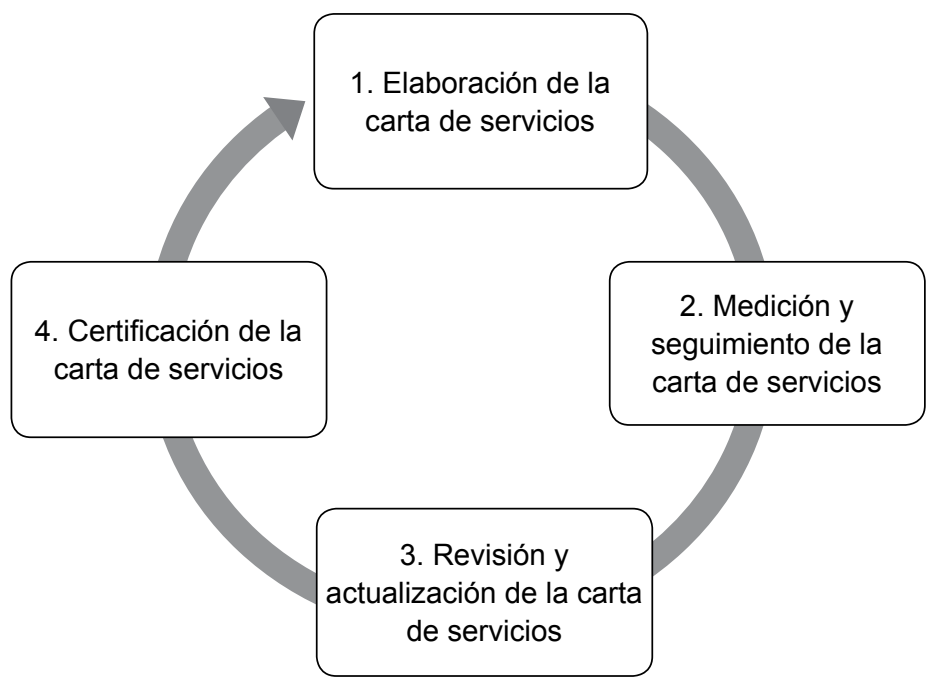

Figura 1. Ciclo para la implementación de cartas de servicios Elaboración propia

La primera etapa se refiere a la elaboración de la carta de servicios, la cual comprende la investigación y análisis de registros de la organización, para a partir de ello fijar indicadores y compromisos orientados a la atención del usuario y que permitan una buena gestión interna. La figura 2 muestra la elaboración de esta carta, desarrollada en nueve pasos.

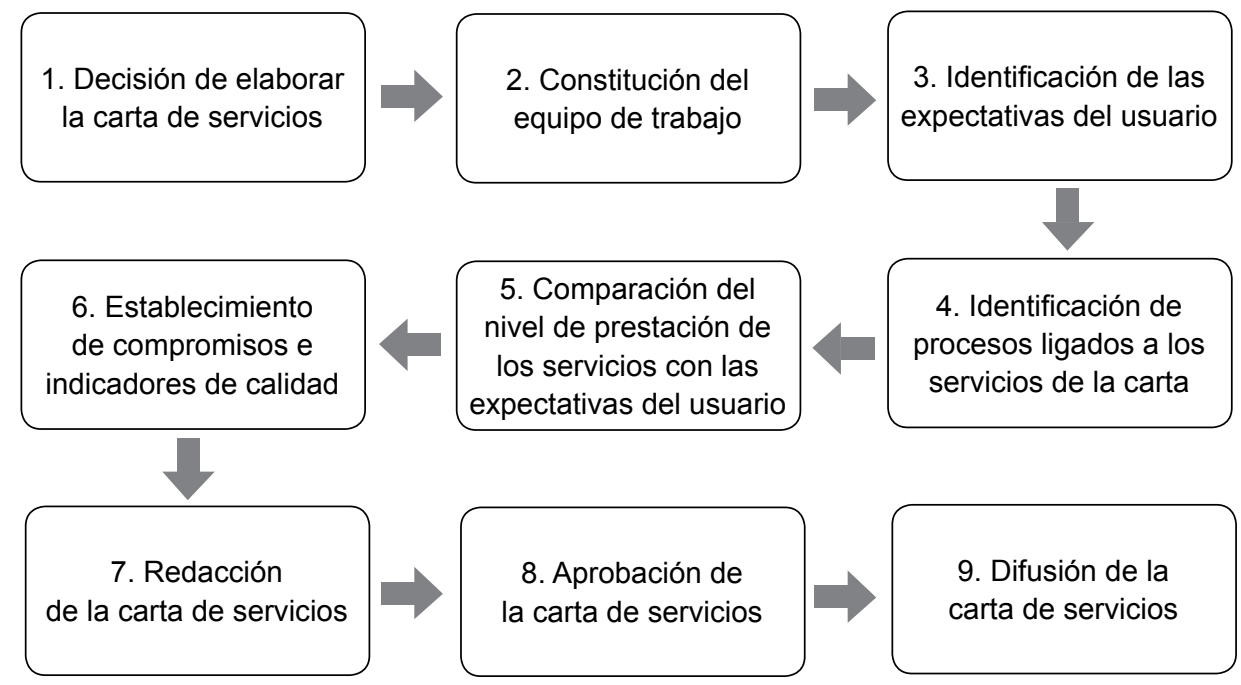

Figura 2. Proceso para la elaboración de cartas de servicios Elaboración propia 
Como todo proceso requiere un punto de partida, el primer paso de esta etapa parte de la formalización y reconocimiento expreso del compromiso de las autoridades y los colaboradores, llamados también agentes de cambio, para emprender el proyecto. Es recomendable registrar el proceso que se optimizará y el compromiso de proporcionar el talento humano y recursos materiales necesarios.

En este sentido, contando con el compromiso formal y el ya identificado proceso de atención que se quiere mejorar, el director del área y su personal más involucrado con dicho proceso deberán formar un equipo de trabajo, el cual necesitará contar con el apoyo y la asesoría de especialistas en materia de gestión por procesos y gestión de calidad (normalmente pertenecientes al área de racionalización o de gestión de calidad de la organización) y continuar la implementación del proyecto, siguiendo los pasos que indica la normativa aplicable ${ }^{3}$.

Posteriormente, el equipo debe recoger las expectativas que el usuario tiene respecto del servicio en particular. Este servicio, como interfaz del proceso que gestionan la institución y el usuario final, será materia de evaluación por parte del usuario, justamente con el fin de descubrir y posteriormente priorizar los factores más valiosos para ellos. Existen diversos métodos para recoger dichas expectativas: encuestas, grupos focales (focus group), paneles y entrevistas. La elección de estos depende de la realidad y masa crítica que desea estudiar la organización. No es casualidad que las expectativas que requiere todo usuario de un servicio recaen en los factores transversales de tiempo, comunicación, forma de atención y respuesta adecuada.

Por otro lado, es importante considerar el nivel de prestación del servicio, esto quiere decir la capacidad que tiene el área para ofrecer un determinado servicio. Para ello es necesario recoger los datos estadísticos correspondientes a los procesos y principales procedimientos mapeados. La dificultad se encuentra en determinar qué variables del proceso dependen del área comprometida y cuáles son controlables internamente.

3 La Norma UNE 93200:2008 dicta los requisitos para la implementación de cartas de servicios, y sugiere de una manera general la metodología para su elaboración, permitiendo libertades en cada paso. A partir de ello, a raíz de experiencias previas y el know how adquirido, las instituciones, como el Ministerio de Trabajo y Promoción del Empleo, pueden desarrollar normas internas para la elaboración e implementación de las cartas de servicios en su organización. 
Cuando se tengan identificadas las expectativas del usuario y el nivel de prestación del servicio, el siguiente paso es comparar ambas fuentes de información bajo una misma perspectiva en particular para cada variable, y en base a ello establecer metas o compromisos de calidad.

Los compromisos pueden ser entendidos como la interfaz que requiere un proceso para gestionar su camino a la mejora continua. Estos deben tener las siguientes características ${ }^{4}$ :

- basados en las expectativas del usuario y la capacidad de la organización

- cuantificables, claros e inequívocos

- deben superar los mínimos normativos

- cada compromiso debe tener como mínimo un indicador

El compromiso, entonces, conforma la esencia de toda carta de servicios, la cual es alcanzada a los usuarios en atención a su búsqueda de un buen servicio.

En consecuencia, la carta de servicios busca formalizar y potenciar los compromisos que asume cada institución a través de dos poderosas vertientes. Primero, busca constituir un claro aporte en la apertura de espacios de participación ciudadana, en donde se priorizan las necesidades y expectativas de los usuarios; y segundo, contribuye en la adaptación y perfeccionamiento de los procesos, lo que deriva en la entrega de bienes y servicios con altos estándares de calidad.

En el marco de esta segunda vertiente, una organización debe asumir compromisos solo en los procesos que generen valor y se encuentren más maduros. Bajo esta premisa, es necesario recopilar información estadística respecto de indicadores de atención existentes, caso contrario es recomendable crear o adaptar las tecnologías para lograr contar con dicho insumo; pero no entendamos esto como una limitante, sino, por el contrario, como el estudio y fortalecimiento de factores internos, y justamente para ello se establecen indicadores de calidad.

Los indicadores de calidad por sí solos suponen una herramienta para medir y hacer seguimiento del comportamiento de ciertas variables propias de un proceso. Estos deben tener las siguientes características:

4 El concepto SMART (por sus siglas en inglés) ayuda a esquematizar estas características, puesto que indica que los objetivos deben ser específicos, medibles, alcanzables, realistas y temporales. 
- se debe definir qué se mide, cómo y cuándo (herramienta $5 \mathrm{~W}+1 \mathrm{H}^{5}$ )

- se deben asignar responsables

- deben ser sensibles

- pueden ser valores absolutos o relativos

La siguiente tabla explica claramente un ejemplo de la caracterización de los indicadores.

Tabla 1.

Caracterización de un indicador de la Carta de Servicios de Absolución Telefónica y Telemática de Consultas Laborales del Ministerio de Trabajo y Promoción del Empleo

\begin{tabular}{|c|c|c|c|c|c|}
\hline $\begin{array}{l}\text { Indicador } \\
\text { (what) }\end{array}$ & $\begin{array}{c}\text { Objetivo } \\
\text { (why) }\end{array}$ & $\begin{array}{c}\text { Responsable } \\
\text { (who) }\end{array}$ & $\begin{array}{c}\text { Frecuencia } \\
\text { (when) }\end{array}$ & $\begin{array}{c}\text { Fórmula } \\
\text { (how) }\end{array}$ & $\begin{array}{l}\text { Medio de } \\
\text { verificación } \\
\text { (where) }\end{array}$ \\
\hline $\begin{array}{l}\text { Porcentaje } \\
\text { de usuarios } \\
\text { satisfechos con } \\
\text { la amabilidad } \\
\text { de la atención } \\
\text { telefónica }\end{array}$ & $>=80 \%$ & $\begin{array}{l}\text { Director de } \\
\text { Capacitación } \\
\text { y Difusión } \\
\text { Laboral del } \\
\text { MTPE }\end{array}$ & $\begin{array}{l}\text { Semanal } \\
\text { (lunes a } \\
\text { viernes) }\end{array}$ & $\begin{array}{l}100 \times \text { [N. }^{\circ} \text { de } \\
\text { usuarios } \\
\text { satisfechos con } \\
\text { la amabilidad } \\
/ \mathrm{N} .^{\circ} \text { total } \\
\text { de usuarios } \\
\text { encuestados] }\end{array}$ & $\begin{array}{l}\text { Sistema } \\
\text { Asternic }\end{array}$ \\
\hline
\end{tabular}

Fuente: Ministerio de Trabajo y Promoción del Empleo

En esta fase del proceso se consigue una visión más amplia del proyecto y se empiezan a vislumbrar ciertos resultados parciales. Por ejemplo, los usuarios se sienten considerados y escuchados al recogerse su opinión a través de encuestas de satisfacción. Por otro lado, el personal operativo o de atención al público, que en un inicio tenía ciertas dudas y era reacio a asumir compromisos, puede ver con más convicción las virtudes que ofrece el nuevo sistema. Para ellos es un sistema con métodos distintos a lo habitual, que implica más carga de trabajo, como llevar más registros y documentar procedimientos, pero entre sus beneficios está el ordenamiento de los procesos, el control de riesgos y la divulgación y exigencia de los requisitos atribuidos al usuario.

5 Herramienta que busca caracterizar un indicador a través de las preguntas ¿qué?, ¿por qué?, ¿dónde?, ¿cuándo?, ¿quién?, ¿cómo? (what?, why?, where?, when?, who?, how?). 
Para culminar con la elaboración de la carta de servicios se debe redactar su contenido final. Es recomendable que para este momento se haya realizado un taller teórico-práctico sobre la implementación de cartas de servicios, o en su defecto, se revise alguna guía metodológica, que además de brindar los conceptos teóricos mínimos que exige la norma, brinde ejemplos y relatos detallados que permitan elaborar un borrador de carta de servicios y demás documentación atribuible a ello. La participación de los funcionarios es importante, pues son ellos quienes toman las decisiones y ejecutan los cambios que se consideren prioritarios, por tal razón estos deben tener pleno conocimiento del tema y encontrarse sensibilizados.

Respecto a la elaboración del documento, una interpretación a grandes rasgos de la información necesaria que debe contener la carta es la siguiente:

\section{A. Información de la organización}

Se refiere a los objetivos y fines de la organización, en donde se identifique el nombre de la institución y las áreas responsables, acompañados de la relación de servicios prestados por estas áreas sobre los cuales se asumirán compromisos. Además, en un apartado se hace referencia a los datos de contacto de la institución, como la persona responsable de la carta, el teléfono de contacto, el correo electrónico, así como la ubicación y los horarios de atención.

También se debe mencionar la relación de la normativa aplicable a los servicios, los derechos y las obligaciones de los usuarios, las formas de acceso y participación de estos, y por último debe mencionarse el documento que aprueba y respalda la carta (puede ser una resolución directoral o de gerencia, según sea el caso), su fecha de vigencia y edición.

\section{B. Compromisos e indicadores}

Debe mostrarse, de forma clara y sin ambigüedades, los compromisos que asume la organización a través de la carta, y los indicadores que permitirán medir el cumplimiento de estos. Es recomendable que una carta no contenga menos de cuatro compromisos.

\section{Mecanismos de comunicación}

Se deben establecer mecanismos eficaces para difundir la carta a toda la organización, por ejemplo a través de reuniones, documentos escritos, periódicos murales o la intranet institucional. 
Asimismo, se deben utilizar canales de comunicación que garanticen la difusión y accesibilidad de la carta a todos los usuarios del servicio, a través de soportes divulgativos como folletos, spots publicitarios o el portal web institucional.

D. Medidas de subsanación

En el caso de que se incumpla algún compromiso de calidad, se deben precisar las medidas que la institución tomará para subsanar esa desviación. Esta subsanación debe estar dirigida al usuario afectado, y servirá de insumo para tomar alguna acción correctiva y preventiva.

E. Presentación de sugerencias, quejas y reclamos

$\mathrm{Al}$ haberse entendido el concepto de estos términos, se hace necesario brindar un espacio al usuario para expresar sus sugerencias, quejas y reclamos respecto del servicio ofrecido, así como al incumplimiento de los compromisos. Deben expresarse las vías para materializar ello.

Todo lo mencionado líneas arriba forma parte del contenido de una carta de servicios. Esta información debe utilizar un lenguaje claro y sencillo (orientado al usuario). A continuación se muestra un modelo de la portada de una carta de servicios.

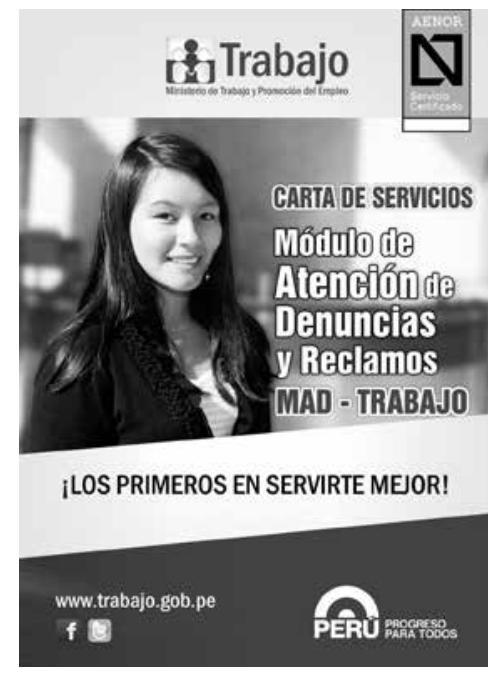

Figura 3. Portada de la carta de servicios del Módulo de Atención de Denuncias y Reclamos del Ministerio de Trabajo y Promoción del Empleo

Fuente: Ministerio de Trabajo y Promoción del Empleo 
Una vez redactada, revisada y validada, la carta de servicios debe ser aprobada por la dirección o autoridad competente, para de esta manera formalizarla y darle un respaldo legal.

Inmediatamente después de su aprobación, la carta de servicios entra en vigencia y se debe realizar su difusión y puesta a disposición de los usuarios y los colaboradores de la institución, a través de los medios establecidos.

La Carta de Servicios, como la que aparece en la figura 3, si bien es el documento que será puesto a disposición del usuario, no es el producto final. Como se mencionó en párrafos anteriores, una finalidad de la carta es servir de instrumento para la gestión interna de los procesos. Para ello, la norma exige que se tenga de respaldo la carta de servicios, un legajo de procedimientos y registros que permitan su medición y seguimiento.

De acuerdo a la figura 4, la conformación de este legajo comprende tres grandes pasos. En primer lugar, es necesario demostrar el mejoramiento de los resultados, para lo cual se procederá a la medición de los indicadores para verificar el cumplimiento de los compromisos, y he aquí la mejora continua, puesto que constantemente el cinturón de la organización se verá ajustado conforme se vayan superando los compromisos en el tiempo.

Es muy importante contar con una herramienta que permita hacer la correcta medición de los indicadores en el transcurso del tiempo. La tabla 2 muestra un buen ejemplo.

Los resultados de la medición de los indicadores deben ser publicados según los formatos que establezca la entidad ${ }^{6}$ y a través de los

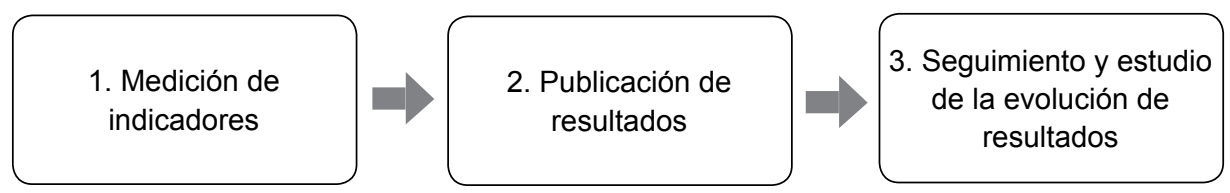

Figura 4. Proceso para la medición y seguimiento de cartas de servicios Elaboración propia

6 Formatos de instituciones como el Ministerio de Trabajo y Promoción del Empleo (MTPE) u organismos públicos como el SAT y el Osinergmin pueden servir de modelos. 
Tabla 2

Medición y seguimiento de indicadores de la carta de servicios de liquidación de beneficios sociales del Ministerio de Trabajo y Promoción del Empleo

\begin{tabular}{|c|c|c|c|c|c|c|c|}
\hline N..$^{\circ}$ & Compromiso & ago-13 & sep-13 & oct-13 & nov-13 & dic-13 & ene-14 \\
\hline \multirow[t]{3}{*}{1} & $\begin{array}{l}\text { Atender al menos al } \\
80 \% \text { de los usuarios que } \\
\text { en el día recabaron un } \\
\text { ticket para el servicio }\end{array}$ & $96 \%$ & $95 \%$ & $92 \%$ & $94 \%$ & $89 \%$ & $95 \%$ \\
\hline & Usuarios derivados & 2289 & 1887 & 1743 & 1390 & 1251 & 2040 \\
\hline & Usuarios atendidos & 2195 & 1788 & 1602 & 1306 & 1114 & 1929 \\
\hline \multirow[t]{2}{*}{2} & $\begin{array}{l}\text { Lograr un nivel de } \\
\text { usuarios satisfechos, } \\
\text { sobre la amabilidad de la } \\
\text { atención brindada, igual } \\
\text { o superior al } 80 \%\end{array}$ & $87 \%$ & $84 \%$ & $84 \%$ & $90 \%$ & $87 \%$ & $95 \%$ \\
\hline & Encuestas realizadas & 58 & 58 & 58 & 26 & 39 & 38 \\
\hline 3 & $\begin{array}{l}\text { Efectuar el cálculo de } \\
\text { la liquidación de los } \\
\text { beneficios sociales } \\
\text { en un tiempo promedio } \\
\text { de } 40 \text { minutos }\end{array}$ & $24 \mathrm{~min}$ & $24 \mathrm{~min}$ & $24 \mathrm{~min}$ & $24 \mathrm{~min}$ & $24 \min$ & $22 \min$ \\
\hline
\end{tabular}

Fuente: Ministerio de Trabajo y Promoción del Empleo

medios de difusión establecidos en la Carta. La puesta a disposición de estos resultados es una prueba de transparencia y compromiso. Sin embargo, el estudio de estos resultados en el tiempo ofrece la posibilidad de gestionar mejoras, para ello se muestra una gráfica de control de indicadores y su evolución en el tiempo.

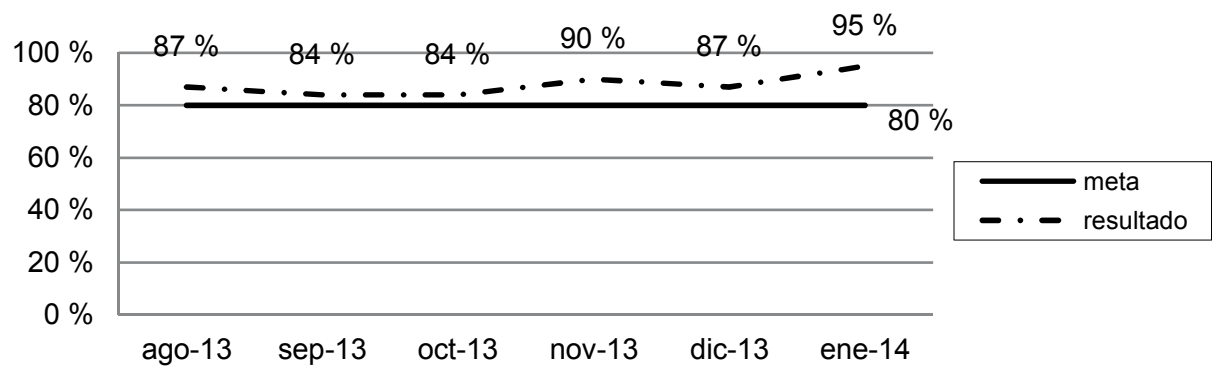

Figura 5. Control de un indicador de la carta de servicios de liquidación de beneficios sociales del Ministerio de Trabajo y Promoción del Empleo

Fuente: Ministerio de Trabajo y Promoción del Empleo 
La carta de servicios no debe ser una finalidad en sí misma, ya que es un instrumento de mejora continua de la calidad de los servicios ofrecidos, que influirá positivamente en la satisfacción de la ciudadanía. Por ello, se hace necesaria su constante revisión y actualización, y para lograrlo se debe considerar en su flujo las acciones correctivas y preventivas y las oportunidades de mejora, conforme se muestran en la figura 6 y explican posteriormente.

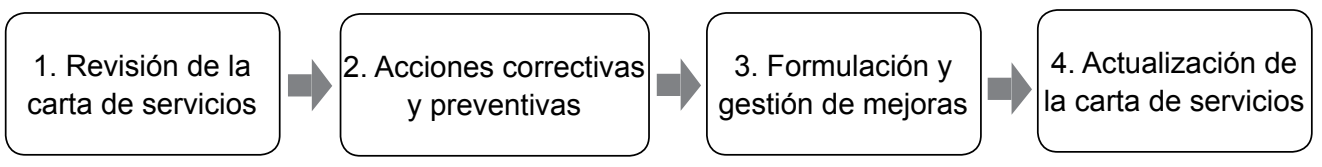

Figura 6. Proceso para la revisión y actualización de cartas de servicios Elaboración propia

En la carta de servicios se establece el período en que esta será revisada (normalmente es anual). Sin embargo, cuando se suscita una oportunidad de mejora o en atención a cambios del entorno que podrían afectar el cumplimiento de los compromisos, su modificación podrá efectuarse antes del plazo establecido.

En la revisión de la carta se identificarán oportunidades de mejora y no conformidades (incumplimiento de los compromisos de calidad) que ameritan un estudio y análisis de causas, de ser el caso. Como en todo tratamiento de desviaciones, estas deben ser canalizadas a través de acciones correctivas y preventivas, planteadas en consenso por el equipo de trabajo.

La gestión de las propuestas de mejora debe realizarse sobre la base de un sustento técnico, para el cual se puede solicitar el apoyo y asesoramiento del comité u oficina de calidad de la institución. Además, debe quedar registrado y a cargo de la dirección administrativa de la organización.

Por último, los cambios y ajustes que resulten de las mejoras gestionadas deberán ser incluidos en una nueva edición de la carta de servicios. Esta debe ser actualizada y se gestionará la modificación del documento legal que la aprobó. Es importante mencionar que la Norma UNE 93200:2008 exige que el proceso de actualización de la carta se base en el que fue empleado para su elaboración. 


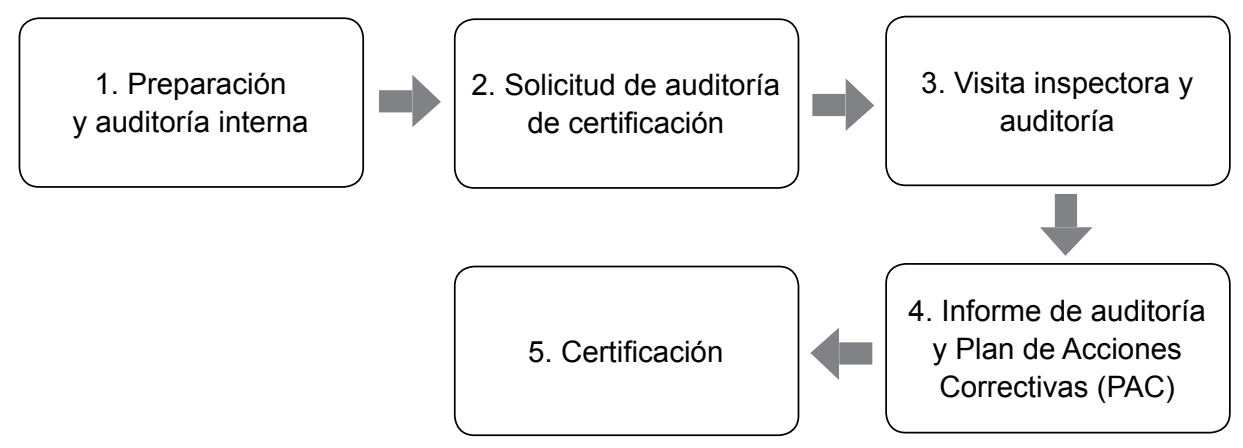

Figura 7. Proceso para la certificación de cartas de servicios Elaboración propia

Hasta este momento se ha descrito los pasos para elaborar una carta de servicios y explicado los mecanismos para su medición, seguimiento, revisión y actualización. Sin embargo, hay un paso más en el camino a la excelencia: la carta de servicios implementada puede ser sometida a los más rigurosos estándares de calidad y reconocida internacionalmente. La validación externa, por parte de una entidad certificadora homologada, aporta un importante valor añadido en cuanto a la fiabilidad y garantía de los compromisos establecidos.

El proceso al que se hace referencia trata de la certificación de la carta de servicios e implica, conforme se expone en la figura 7 , una preparación a través de una auditoría interna, seguida de una auditoría externa, la subsanación de no conformidades, de ser el caso, y el informe final de aprobación y concesión de la marca de certificación.

Para explicar cada subproceso descrito previamente, es importante conocer que el proceso de implementación de la carta de servicios será evaluado bajo la Norma UNE 93200:2008 "Cartas de Servicios", que ha sido elaborada en España por el comité técnico AEN/CTN 93, bajo la secretaría técnica de la Asociación Española de Normalización y Certificación AENOR, y que en la actualidad es materia de supervisión en el Perú.

El procedimiento de certificación consta de una visita inspectora a través de un cliente misterioso ${ }^{7}$, seguida de una auditoría in situ al

$7 \quad$ El cliente misterioso o cliente incógnito es una técnica utilizada por las empresas para evaluar y medir la calidad en la atención al cliente o usuario. Los clientes 
servicio postulante. Al igual que en un sistema de gestión, se verifica el cumplimiento de la norma y en caso de encontrar no conformidades se solicita el plan de acciones correctivas, que de ser idóneo no da lugar más que a la obtención del anhelado certificado.

A modo de preparación para la auditoría de certificación, y en forma complementaria, se sugiere la realización de una auditoría interna. Este tipo de auditoría sigue la misma metodología que las auditorías externas, debe ser realizada por profesionales capacitados en auditorías internas que la institución autorice y siguiendo un procedimiento establecido por esta. La entidad formará a los auditores internos, los cuales deben ejercer con el asesoramiento del área de calidad correspondiente de la entidad. Un auditor interno no puede auditar el área donde trabaja.

Por último, es importante mencionar que después de la certificación del servicio postulante, la empresa normalizadora establece un período de seguimiento, durante el cual se deben realizar auditorías de seguimiento por el lapso de vigencia del certificado (usualmente anual).

\section{DISCUSIÓN}

Ahora bien, en este momento el lector, ya sea un universitario con interés en temas de calidad o un jefe de oficina con un proceso a su cargo próximo a seguir un modelo de mejora, debe estar en capacidad de responderse las siguientes preguntas:

- ¿Por qué es importante la carta de servicios como una herramienta de mejora continua?

- ¿Qué ventajas tiene esta herramienta respecto de otras?

- ¿Cuál es la finalidad de la carta de servicios y cuáles son sus dos principales vertientes?

- ¿Qué significa asumir un compromiso y de qué manera a través de este se gestiona el camino a la excelencia?

Estas preguntas solo buscan reconocer el sentido de una carta de servicios y su filosofía bajo el contexto de una cultura de calidad. Al cuestionarse ello, hay que alinear sus metas a las estrategias de la or-

misteriosos actúan como clientes comunes que realizan una compra o consumen un servicio, luego lo evalúan y entregan un informe acerca de su experiencia. 
ganización donde laboramos, con el fin de encaminarlas a sumar como una herramienta para lograr la excelencia.

Esta herramienta, y la Norma UNE 93200:2008 que lo respalda, expone un proyecto innovador, económico y fácil, con mucha acogida y de alcance internacional, que logra resultados a corto plazo en una institución pública como privada. Con ánimos de llegar más allá, la carta de servicios ha significado una excelente herramienta en la búsqueda de una cultura de calidad. Esto se explica mejor a través de la siguiente tabla comparativa, donde se aprecian los cambios significativos en una organización, derivados de su implementación.

Tabla 3

Beneficios obtenidos con las cartas de servicios en el Ministerio de Trabajo y Promoción del Empleo

\begin{tabular}{|c|c|}
\hline Año 2012 & Año 2014 \\
\hline Resistencia al cambio & Reducción de la resistencia al cambio \\
\hline $\begin{array}{l}\text { Ausencia de conciencia de la } \\
\text { calidad en el servicio al ciudadano }\end{array}$ & $\begin{array}{l}\text { Mayor conocimiento de la calidad en el servicio } \\
\text { al ciudadano }\end{array}$ \\
\hline $\begin{array}{l}\text { Carencia de encuestas } \\
\text { de necesidades y expectativas } \\
\text { del usuario }\end{array}$ & $\begin{array}{l}\text { Se realizan encuestas de necesidades por cada } \\
\text { carta de servicios. Existen cuatro expectativas } \\
\text { generales en todo servicio: tiempo, forma de } \\
\text { atención, respuesta confiable y comunicación }\end{array}$ \\
\hline $\begin{array}{l}\text { Carencia de encuestas } \\
\text { de satisfacción }\end{array}$ & $\begin{array}{l}\text { Existen encuestas de satisfacción por cada carta } \\
\text { de servicios }\end{array}$ \\
\hline $\begin{array}{l}\text { Poco apoyo a los comités y grupos } \\
\text { de mejora }\end{array}$ & $\begin{array}{l}\text { Los grupos de mejora se están integrando en la } \\
\text { gestión }\end{array}$ \\
\hline Poca comunicación entre áreas & Integración de las áreas con un objetivo común \\
\hline Trabajo aislado & Trabajo en equipo \\
\hline Trabajo por funciones (vertical) & Trabajo por procesos (horizontal) \\
\hline
\end{tabular}

Fuente: Ministerio de Trabajo y Promoción del Empleo

Como estrategas y gestores de cambio, nos interesan los beneficios de esta herramienta y más aún si tenemos un plan estratégico de largo plazo. Por otro lado, al usuario le interesan los resultados concretos, la buena atención, recibir un servicio de calidad, un servicio A-1, y si bien ello es poco creíble para algunos que por cuestiones y experiencias 
particulares difieren de la realidad, lo que corresponde hacer a la organización es reavivar el compromiso, romper esquemas, y demostrar que está comprometida y apunta a la excelencia.

Finalmente, conociendo los conceptos básicos sobre cartas de servicios, los requisitos que exige la norma y el procedimiento que se debe seguir para su implementación y certificación, no queda más que citar ciertas conclusiones a las que llegué después de aplicar este modelo en una institución del Estado peruano; para ser más específico, en el Ministerio de Trabajo y Promoción del Empleo (MTPE), la entidad protagonista de las deficiencias que evidencia el Estado y que han sido mencionadas al inicio del presente artículo; de ahí mis conclusiones y recomendaciones.

\section{CONCLUSIONES}

Teniendo en cuenta que la prestación de servicios al público es la imagen de una organización, es importante que el personal comprenda el concepto que se tiene por calidad en el servicio, para ello la carta de servicios resulta un instrumento importante para alcanzar dicho objetivo.

- La carta de servicios recoge varias técnicas de ingeniería industrial en su desarrollo para mapear los procesos, hacer el análisis de riesgo, determinar las causas raíz, proponer las soluciones y analizar la relación beneficio/costo, entre otras.

- Este proyecto ha sido implementado en el MTPE y ha tenido un gran impacto en la satisfacción del usuario y en el clima laboral:

- Se pasó de un $87 \%$ a un $95 \%$ de satisfacción del usuario en menos de seis meses.

- Se logró incrementar a 76,8 \% en la satisfacción de los colaboradores.

- Se cuenta con un Plan de Desarrollo de las Personas, que busca el reconocimiento y la retención del talento del MTPE.

- Asimismo, se tiene un Plan de Desarrollo y Cultura de la Calidad, dirigido a las autoridades y personal en general del MTPE.

- Las mejoras realizadas en el desarrollo de esta metodología implican la estandarización de procedimientos y generación de nuevos indicadores de calidad. Asimismo, estos indicadores, convertidos en compromisos, se pueden normalizar y certificar bajo el ámbito de la norma española UNE 93200:2008.

- Gracias a la implementación de la Carta de Servicios y a los resultados positivos logrados con la mejora de los procesos de atención, 
diferentes áreas del MTPE se han contagiado de esta buena práctica y se vienen sumando al proceso de estandarización. Esto también ha derivado en la aprobación de una "Guía metodológica para la implementación de cartas de servicios en el Ministerio de Trabajo y Promoción del Empleo", la cual se encuentra disponible en el portal institucional de este organismo estatal (www.trabajo.gob.pe).

- Otro aspecto importante de esta herramienta radica en que formará parte del Sistema de Gestión de Calidad, ya que la carta de servicios mantiene todas las características y requisitos previstos en la Norma ISO 9001:2008, constituyendo un factor determinante para su adopción.

\section{RECOMENDACIONES}

- Si bien la carta de servicios es un instrumento que ayuda a controlar los procesos de manera ágil y orientada al usuario, es de mucha ayuda que los colaboradores tengan una noción previa del concepto de gestión por procesos. De la misma manera, se recomienda capacitar al personal sobre la atención de calidad al usuario, ya que ello permitirá dar sostenibilidad a lo logrado.

- Ampliar el alcance de aplicación de las cartas de servicios y considerar los demás procesos críticos de atención al usuario, para así crear un compromiso institucional.

- Programar oportunamente el presupuesto destinado a las actividades comprendidas en la gestión de la calidad, puesto que cualquier retraso o inconveniente afectará indirectamente al usuario, además de implicar gastos adicionales. Asimismo, se sugiere contar con el respaldo de la alta dirección o gerencia de la institución, que impulse y brinde el apoyo para la ejecución de esta estrategia.

- Que los responsables de los procesos otorguen sostenibilidad a las cartas de servicios aprobadas y que se empodere y delegue a la oficina o comité de gestión de calidad de la institución el monitoreo y seguimiento de estas, e impulse la implementación y certificación de nuevas cartas.

- Finalmente, que la experiencia en la implementación de cartas de servicios sirva para promover la calidad en la cultura organizacional de la institución. 


\section{REFERENCIAS}

Bonilla, E., Díaz, B., Kleeberg, F., y Noriega, M. T. (2010). Mejora continua de los procesos: Herramientas y técnicas. Lima: Universidad de Lima.

Ministerio de Trabajo y Promoción del Empleo. (2013). Primeros en cartas de servicios. Recuperado de http://trabajo.gob.pe.

Ministerio de Trabajo y Promoción del Empleo. (2015). Resolución del Secretario General N. ${ }^{\circ}$ 126-2013-TR/SG, que aprueba la "Guía para la implementación de Cartas de Servicios en el Ministerio de Trabajo y Promoción del Empleo". Recuperado de http://trabajo.gob.pe.

Presidencia del Consejo de Ministros. (2013). Resolución Ministerial N. ${ }^{\circ}$ 156-2013-PCM, que aprueba el "Manual para Mejorar la Atención a la Ciudadanía en las entidades de la Administración Pública". Diario oficial El Peruano. Recuperado de http://www.munizlaw. com/normas/2013/Junio/19-06-13/R.M.\%20N\%C2\%BA\%201562013-PCM.pdf

Rodríguez, J., y Ugarte, J. (2014). Casos prácticos: Ministerio de Trabajo de Perú, primeros en cartas de servicios. AENOR. Revista de la Normalización y la Certificación, 289, 30.

Secretaría de Gestión Pública (SGP) - Presidencia del Consejo de Ministros. (2012). Estrategia de modernización. Recuperado de http://sgp.pcm.gob.pe

Sociedad Española de Normalización y Certificación. (Abril de 2008). Norma Española UNE 93200: 2008 "Cartas de Servicios" Requisitos. AENOR, 6 .

Ugarte, J. (2014). Mejora de los procesos de atención en el área de la SubDirección de Defensa Legal Gratuita y Asesoría al Trabajador del Ministerio de Trabajo y Promoción del Empleo. (Tesis para obtener el título de ingeniero industrial). Universidad de Lima, Perú. 